\title{
The Mu2e Solenoid Cold Mass Position Monitor System
}

\section{The Mu2e Experiment}

The Mu2e experiment at Fermilab is designed to search for charged-lepton flavor violation by searching for neutrinoless muon to electron conversions in the field of a target nucleus. The concept of the experiment is to generate a low momentum muon beam, stopping the muons in a target and measuring the momentum of the conversion electrons.

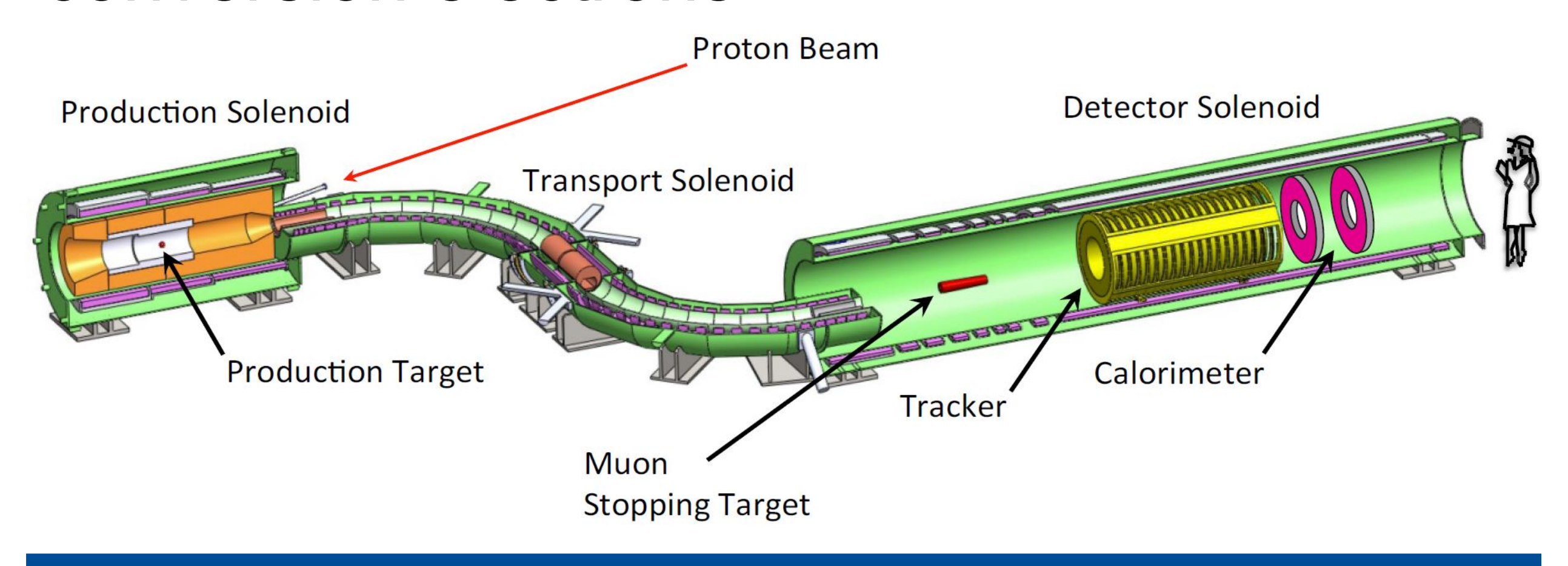

\section{The Mu2e Solenoids}

Three different solenoids create a complex magnetic field with graded solenoidal and toroidal components to guide muons created in the Production Target to the Muon Stopping Target. The magnet coils will operate at $4.5 \mathrm{~K}$ and be shielded inside a cryostat. During cool down and magnet excitation the cold masses will move up to $37 \mathrm{~mm}$ with respect to the outside cryostat fiducials.

$$
\begin{aligned}
& \text { Further information is available at: } \\
& \text { [1] thpp://mu2.efnal. gov } \\
& \text { cal Design Report, arXiv:1501.05241 }
\end{aligned}
$$

\section{The Solenoid Alignment}

Monitoring coil movements relative to the external fiducials is required for alignment of the solenoids and to predict shifts of the magnetic field. A high precision magnet field map is taken for the Detector Solenoid required for the momentum reconstruction of the conversion electrons. Small solenoid movements will result in changes of the magnetic field in the tracker, affecting the resolution or introducing systematic calibration errors.

\section{The Cold Mass Position Monitoring System}

Mu2e needs to know the position of the Detector Solenoid Cold Mass with a precision better than 1 $\mathrm{mm}$. The system will need to work remotely, as the cryostat is inaccessible. It has to seal against vacuum better than $10^{-7}$ torr and withstand overpressure of $15 \mathrm{psi}$, and survive a dose of $1 \mathrm{kGray} / \mathrm{year}$ The system has to work for the $\sim 3$ year lifetime of the experiment in a magnetic field.

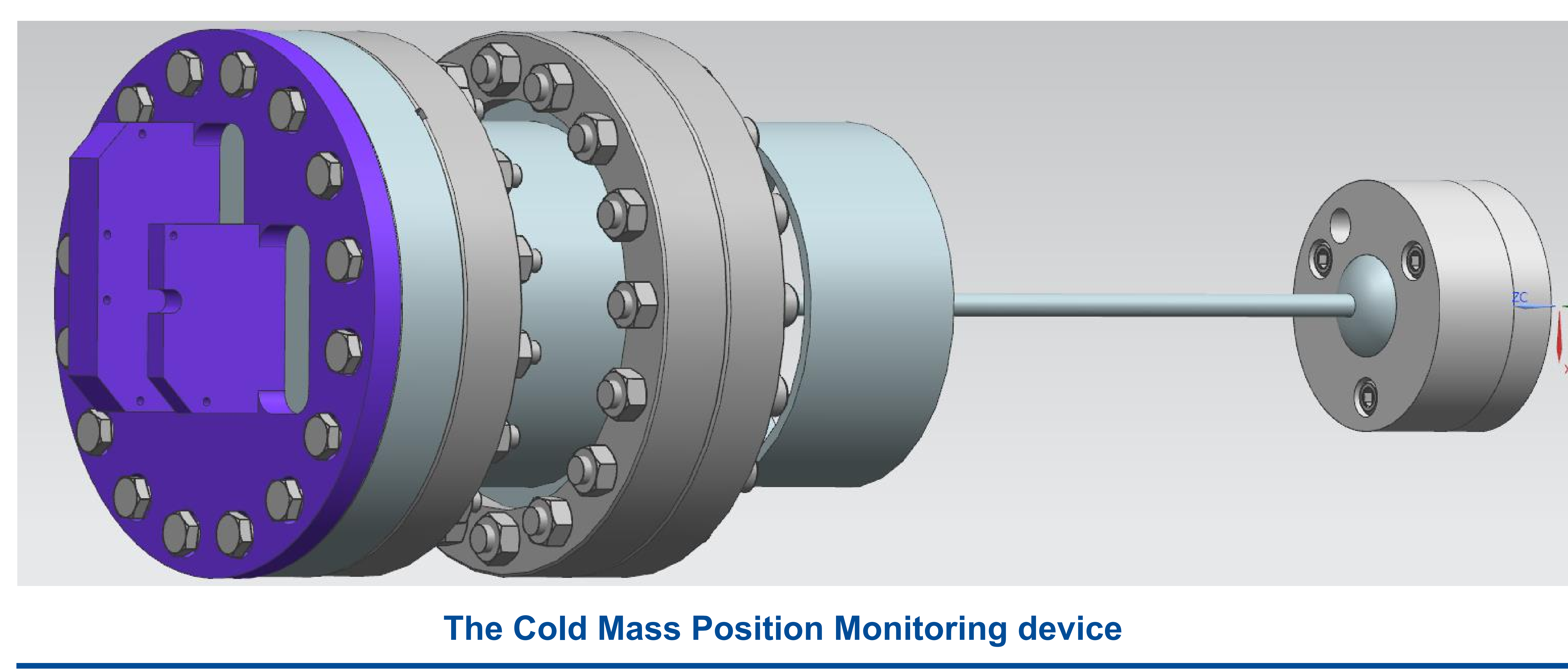

\section{The Components}
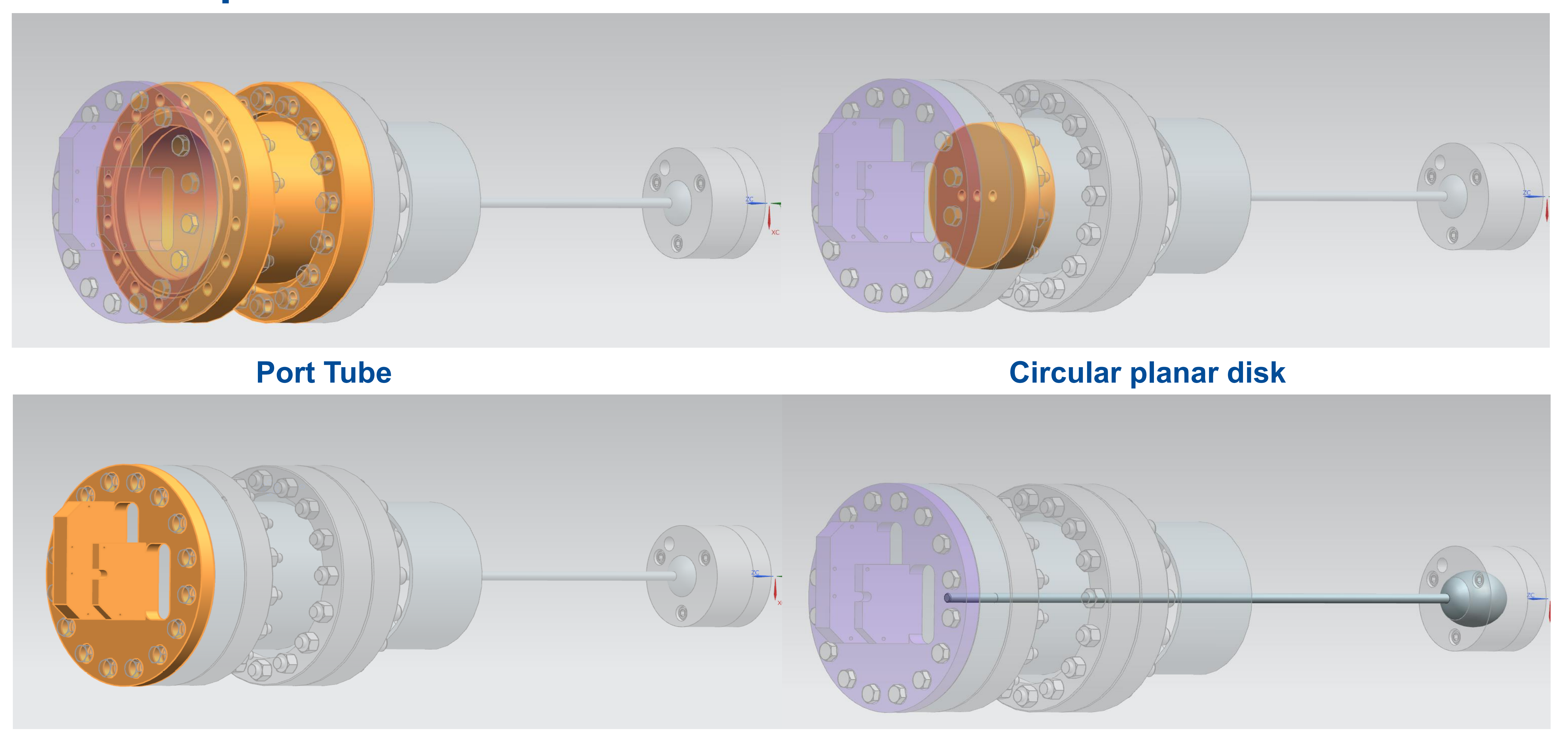

Laser Sensor Mounting plate
The Measurement principle

The solenoid is a rigid body and we survey 4 locations to measure its movements. At each survey point, a spherical mount is affixed to the Cold Mass. A second circular planar disk moving freely in a port tube is connected to the cold mass via a fixed length rod. Three Keyence IL-065 laser sensors, with $45 \mathrm{~mm}$ range and 2 micrometer accuracy, are mounted on a plate fitted to a Quartz vacuum window. We measure distances between the mounting plate and disk along calibrated laser paths. The angle of the port tube axis w.r.t. the sensor plate is surveyed, and thus we translate the disk movement into the displacement of the sphere affixed to the Cold Mass.

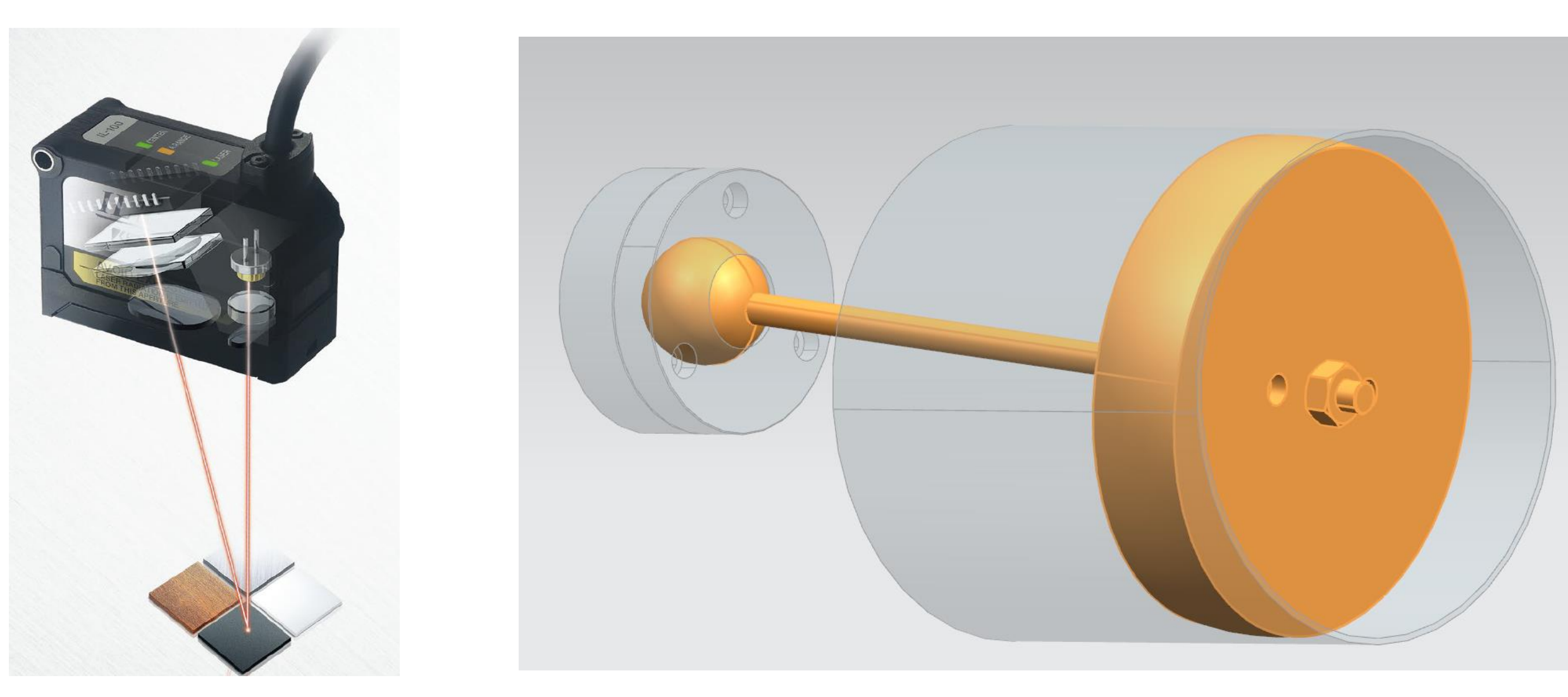

Keyence IL-065 Visualization of the disk tilt when laser sensor the sphere moves

\section{The expected resolution}

We will calibrate the path of the Keyence IL065 laser sensors after affixing them to the mounting plate. This results in a readback error of less than 2 microns for the IL-065 (plus 2 microns syst.). Assuming a $10 \mathrm{~cm}$ lever rod the geometrical displacement of the sphere thus has a position error of 20 microns. The port tube and sensor plate will be aligned via CMM to 25 microns, the length of the rod is known better than 25 microns too. In total we expect the error on the cold mass reading to be less than 100 microns in three dimensions. 